\title{
函数 S-粗集与规律辨识
}

\section{史开泉 ${ }^{\mathbb{Q} * *}$, 姚炳学}

(1) 山东大学数学与系统科学学院, 济南 250100;

(2) 聊城大学数学科学学院, 聊城 252059

*E-mail: shikq@sdu.edu.cn

收稿日期: 2006-10-16; 接受日期: 2007-09-24

国家自然科学基金(批准号:60364001)、教育部科学技术研究重点项目(批准号：206089)和山东省自然科学基金(批准 号: Y2007H02)资助项目

摘要 把具有动态特征的元素等价类, 引入到 Pawlak Z 粗集中, 对 Pawlak $\mathrm{Z}$ 粗集给出改进, 提出 S-粗集(singular rough sets), S-粗集是用具有动态特性 的元素等价类定义的, S-粗集具有动态特性. 把具有动态特性的函数等价类 (规律等价类), 引入到 S-粗集中, 对 S-粗集给出改进, 提出函数 S-粗集 (function singular rough sets), 函数 S-粗集具有了动态特性和规律特性. 一个 函数是一个规律. 利用函数 S-粗集, 给出规律辨识、规律辨识定理、规律辨

关键词

函数 S-粗集

系统规律

规律辨识

辨识准则

辨识定理 识准则与应用. 函数 $\mathrm{S}$-粗集是粗集理论的一个新的研究方向, 函数 S-粗集是 系统规律辨识研究的一个新的工具.

多端输出的信息系统或者单端输出的信息系统, 在其工作的时间区间 $T_{j}=\left[t_{1}, t_{k}\right]$ 上, 都要 对其输出规律状态进行辨识, 以此确认 $T_{j}=\left[t_{1}, t_{k}\right](j=1,2, \cdots, \lambda)$ 上信息系统的输出规律: 规律 稳定或者规律紊乱. $m$ 端输出的信息系统, 在 $T_{j}=\left[t_{1}, t_{k}\right]$ 上的输出 $u_{1}, u_{2}, \cdots, u_{m}$ 构成了关于 $T_{j}=\left[t_{1}, t_{k}\right]$ 的函数等价类 ${ }^{[1,2]}$ (规律等价类) $[u]_{T_{j}}=\left\{u_{1}, u_{2}, \cdots, u_{m}\right\}, u_{i} \in[u]_{T_{j}}$ 是一个函数, 函数是 一个规律; 在 $t_{\lambda} \in T_{j}$ 上的输出 $u_{1}\left(t_{\lambda}\right), u_{2}\left(t_{\lambda}\right), \cdots, u_{m}\left(t_{\lambda}\right)$ 构成了关于 $t_{\lambda} \in T_{j}$ 的元素等价类 $\left(t_{\lambda}\right.$ 点的 规律状态值) $[x]_{t_{\lambda}}=\left\{u_{1}\left(t_{\lambda}\right), u_{2}\left(t_{\lambda}\right), \cdots, u_{m}\left(t_{\lambda}\right)\right\}=\left\{x_{1}, x_{2}, \cdots, x_{m}\right\}$.

文献 [1,2]给出 $R$-函数等价类和函数迁移的概念; 利用这些概念, 文献 [1,2]提出函数 S-粗 集(function singular rough sets), 给出了它的 3 类形式; 提出函数粗集(function rough sets). 函数 粗集是函数 S-粗集的简化形式与静态形式; 文献 [3 9]对函数 S-粗集的理论与应用给出了进一 步讨论.

因为函数 S-粗集具有动态特征和规律特征，与信息系统中的信息规律辨识之间存在着交 
叉和渗透点; 本文把函数 S-粗集与信息系统中的信息规律辨识进行交叉、嫁接, 给出信息系统 中的信息规律辨识、规律辨识准则、规律辨识定理与应用. 函数 S-粗集与信息系统中的信息规 律辨识的交叉、渗透、嫁接，是信息系统中信息规律辨识研究的一个新方向.

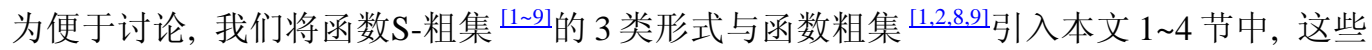
概念, 对于规律辨识研究是重要的.

约定 $\mathscr{O}(x)$ 是有限函数论域; $Q(x) \subset \mathscr{O}(x)$ 是有限函数集; $F=\left\{f_{1}, f_{2}, \cdots, f_{m}\right\}, \bar{F}=$ $\left\{\bar{f}_{1}, \bar{f}_{2}, \cdots, \bar{f}_{n}\right\}$ 是 $\mathscr{D}(x)$ 上的函数迁移族; $[u(x)]=\left\{u_{1}(x), u_{2}(x), \cdots, u_{n}(x)\right\}$ 是 $R$-函数等价类; $R$ 是等 价关系; $(x), Q(x),[u(x)], u(x), v(x)$ 分别记作 $\mathscr{O}, Q,[u], u, v$. 本文 $1 \sim 4$ 节中出现的概 念, 在文献 [1 14]中都能找到.

\section{1 函数单向 S-粗集}

$Q^{\circ}$ 是 $Q \subset \mathscr{D}$ 的单向 S-函数集合(one direction singular function sets), 如果

$$
Q^{\circ}=Q \bigcup\{v \mid v \in \mathscr{R}, v \bar{\in} Q, f(v)=u \in Q\} .
$$

$Q^{f}$ 是 $Q$ 的 $f$-扩张, 如果

$$
Q^{f}=\{v \mid v \in \mathscr{D}, v \bar{\in} Q, f(v)=u \in Q\},
$$

这里 $f \in F$ 是函数迁移 ${ }^{[1,2,8,9]}, f \in F$ 的作用是: $v \in \mathscr{D}, v \in Q, f \in F$ 把 $v$ 变成 $f(v)=u \in Q$. $(R, F) \circ\left(Q^{\circ}\right)$ 和 $(R, F)^{\circ}\left(Q^{\circ}\right)$ 分别是 $Q^{\circ} \subset \mathcal{O}$ 的下近似和上近似, 如果

$$
\begin{aligned}
(R, F) \circ\left(Q^{\circ}\right) & =\bigcup[u] \\
& =\left\{u \mid u \in \mathscr{D},[u] \subseteq Q^{\circ}\right\}, \\
(R, F)^{\circ}\left(Q^{\circ}\right)= & \bigcup[u] \\
= & \left\{u \mid u \in \mathscr{D},[u] \cap Q^{\circ} \neq \varnothing\right\} .
\end{aligned}
$$

$B_{n R}\left(Q^{\circ}\right)$ 是 $Q^{\circ} \subset \mathscr{D}$ 的 $R$-边界, 如果

$$
B_{n R}\left(Q^{\circ}\right)=(R, F)^{\circ}\left(Q^{\circ}\right)-(R, F) \circ\left(Q^{\circ}\right),
$$

这里 $F \neq \varnothing$.

$(R, F) \circ\left(Q^{\circ}\right)$ 和 $(R, F)^{\circ}\left(Q^{\circ}\right)$ 构成的集合对是 $Q^{\circ} \subset \mathscr{O}$ 的函数单向 S-粗集 (function one direction singular rough sets), 如果

$$
\left((R, F) \circ\left(Q^{\circ}\right),(R, F)^{\circ}\left(Q^{\circ}\right)\right) .
$$

$A s\left(Q^{\circ}\right)$ 是函数单向 $\mathrm{S}$-粗集生成的副集(assistant sets), 如果

$$
A s\left(Q^{\circ}\right)=\{v \mid v \in \mathscr{D}, v \bar{\in} Q, f(v)=u \tilde{\in} Q\} .
$$

这里 $A s\left(Q^{\circ}\right)$ 中的 “ $\tilde{\epsilon}$ ”表示元素 $f(v)=u$ 与集合 $Q$ 的关系满足特征函数 $0<\chi_{Q}^{f(v)}<1$; (2)式中的 元素 $f(v)=u$ 与集合 $Q$ 的关系满足特征函数 $\chi_{Q}^{f(v)}=1$. 


\section{2 函数双向 S-粗集}

$Q^{*}$ 是 $Q \subset \mathscr{D}$ 的双向 S-函数集合(two direction singular function sets), 如果

$$
\begin{gathered}
Q^{*}=Q^{\prime} \bigcup\{v \mid v \in \mathscr{Q}, v \bar{\in} Q, f(v)=u \in Q\}, \\
Q^{\prime}=Q-\{u \mid u \in Q, \bar{f}(u)=v \bar{\in} Q\} .
\end{gathered}
$$

$Q^{\bar{f}}$ 是 $Q \subset \mathscr{O}$ 的 $\bar{f}$-萎缩, 如果

$$
Q^{\bar{f}}=\{u \mid u \in Q, \bar{f}(u)=v \bar{\in} Q\},
$$

这里 $\bar{f} \in \bar{F}$ 是函数迁移 ${ }^{[1,2,8,9]}, \bar{f} \in \bar{F}$ 的作用是: $u \in Q$ 和 $\bar{f} \in \bar{F}$ 把 $u$ 变成 $\bar{f}(u)=v \bar{\in} Q$. $(R, \mathscr{F}) \circ\left(Q^{*}\right)$ 和 $(R, \mathscr{F})^{\circ}\left(Q^{*}\right)$ 分别是 $Q^{*} \subset \mathscr{O}$ 的下近似和上近似, 如果

$$
\begin{aligned}
(R, \mathscr{F}) \circ\left(Q^{*}\right) & =\bigcup[u] \\
& =\left\{u \mid u \in \mathscr{O},[u] \subseteq Q^{*}\right\}, \\
(R, \mathscr{F})^{\circ}\left(Q^{*}\right)= & \bigcup[u] \\
= & \left\{u \mid u \in \mathscr{O},[u] \cap Q^{*} \neq \varnothing\right\} .
\end{aligned}
$$

$B_{n R}\left(Q^{*}\right)$ 是 $Q^{*} \subset \mathcal{O}$ 的 $R$-边界, 如果

$$
B_{n R}\left(Q^{*}\right)=(R, \mathscr{F})^{\circ}\left(Q^{*}\right)-(R, \mathscr{F}) \circ\left(Q^{*}\right),
$$

这里 $\mathscr{F}=F \cup \bar{F}, F \neq \varnothing, \bar{F} \neq \varnothing$.

$(R, \mathscr{F}) \circ\left(Q^{*}\right)$ 和 $(R, \mathscr{F})^{\circ}\left(Q^{*}\right)$ 构成的集合对是 $Q^{*} \subset \mathscr{O}$ 的函数双向 S-粗集(function two direction singular rough sets), 如果

$$
\left((R, \mathscr{F}) \circ\left(Q^{*}\right),(R, \mathscr{F})^{\circ}\left(Q^{*}\right)\right) .
$$

$\operatorname{As}\left(Q^{*}\right)$ 是函数双向 $\mathrm{S}$-粗集生成的副集, 如果

$$
A s\left(Q^{*}\right)=\{u \mid v \in \mathscr{O}, v \bar{\in} Q, f(v)=u \tilde{\in} Q \text { 和 } u \in Q, \bar{f}(u)=v \in Q\},
$$

这里: $A s\left(Q^{*}\right)$ 中的 “ $\Subset$ ”表示元素 $\bar{f}(u)=v$ 与集合 $Q$ 的关系满足特征函数 $-1<\chi_{Q}^{\bar{f}(u)}<0 ;(10)$ 式 中的元素 $\bar{f}(u)=v$ 与集合 $Q$ 的关系满足特征函数 $\chi_{Q}^{\bar{f}(u)}=-1$.

\section{3 函数单向 S-粗集对偶}

$Q^{\prime} \subset \mathscr{O}$ 是单向 S-函数集合 $Q^{\circ}=Q \bigcup\{v \mid v \in \mathscr{O}, v \bar{\in} Q, f(v)=u \in Q\}$ 的对偶, 如果

$$
Q^{\prime}=Q-\{u \mid u \in Q, \bar{f}(u)=v \bar{\in} Q\} .
$$

$(R, \bar{F}) \circ\left(Q^{\prime}\right)$ 和 $(R, \bar{F})^{\circ}\left(Q^{\prime}\right)$ 分别是 $Q^{\prime} \subset \mathcal{O}$ 的下近似和上近似，如果

$$
\begin{aligned}
(R, \bar{F}) \circ\left(Q^{\prime}\right) & =\bigcup[u] \\
& =\left\{u \mid u \in \mathscr{O},[u] \subseteq Q^{\prime}\right\},
\end{aligned}
$$




$$
\begin{aligned}
(R, \bar{F})^{\circ}\left(Q^{\prime}\right) & =\bigcup[u] \\
& =\left\{u \mid u \in \mathscr{O},[u] \cap Q^{\prime} \neq \varnothing\right\} .
\end{aligned}
$$

$B_{n R}\left(Q^{\prime}\right)$ 是 $Q^{\prime} \subset \mathscr{O}$ 的 $R$-边界, 如果

$$
B_{n R}\left(Q^{\prime}\right)=(R, \bar{F})^{\circ}\left(Q^{\prime}\right)-(R, \bar{F})_{\circ}\left(Q^{\prime}\right),
$$

这里 $\bar{F} \neq \varnothing$.

$(R, \bar{F}) \circ\left(Q^{\prime}\right)$ 和 $(R, \bar{F})^{\circ}\left(Q^{\prime}\right)$ 构成的集合对是 $Q^{\prime} \subset \mathscr{O}$ 的函数单向 S-粗集对偶(dual of function one direction singular rough sets), 如果

$$
\left((R, \bar{F}) \circ\left(Q^{\prime}\right),(R, \bar{F})^{\circ}\left(Q^{\prime}\right)\right) .
$$

$\operatorname{As}\left(Q^{\prime}\right)$ 是函数单向 $\mathrm{S}$-粗集对偶生成的副集, 如果

$$
A s\left(Q^{\prime}\right)=\{u \mid u \in Q, \bar{f}(u)=v \in Q\} .
$$

\section{4 函数粗集}

对于第 2 节中的结果, 若函数迁移族 $F=\varnothing, \bar{F}=\varnothing, \mathscr{F}=F \cup \bar{F}=\varnothing$; 则(8)和(9)式中的 $\{v \mid v \in \mathscr{D}, v \bar{\in} Q, f(v)=u \in Q\}=\varnothing,\{u \mid u \in Q, \bar{f}(u)=v \bar{\in} Q\}=\varnothing$; (8)和(9)二式成为 $Q^{*}=Q^{\prime}=Q$, 则有 $Q \subset \mathscr{O}$ 的下近似 $R_{-}(Q)$ 和上近似 $R^{-}(Q)$, 如果

$$
\begin{aligned}
R_{-}(Q) & =\bigcup[u] \\
& =\{u \mid u \in \mathscr{O}),[u] \subseteq Q\}, \\
R^{-}(Q)= & \bigcup[u] \\
= & \{u \mid u \in \mathscr{O},[u] \cap Q \neq \varnothing\} .
\end{aligned}
$$

$B_{n R}(Q)$ 是 $Q \subset \mathscr{O}$ 的 $R-$ 边界, 如果

$$
B_{n R}(Q)=R^{-}(Q)-R_{-}(Q) .
$$

$R_{-}(Q)$ 和 $R^{-}(Q)$ 构成的集合对是 $Q \subset \mathscr{O}$ 的函数粗集，如果

$$
\left(R_{-}(Q), R^{-}(Q)\right) .
$$

利用 $1 \sim 4$ 节的结果, 容易证明定理 1 5 和推论 1 3.

定理 1 函数双向 $\mathrm{S}$-粗集与函数单向 $\mathrm{S}$-粗集满足

$$
\left((R, \mathscr{F}) \circ\left(Q^{*}\right),(R, \mathscr{F})^{\circ}\left(Q^{*}\right)\right)_{\bar{F}=\varnothing}=\left((R, F) \circ\left(Q^{\circ}\right),(R, F)^{\circ}\left(Q^{\circ}\right)\right) \text {. }
$$

证明 若 $\bar{F}=\varnothing, \mathscr{F}=F \cup \bar{F}$ 成为 $\mathscr{F}=F$; 则(10)式中 $Q^{\bar{f}}=\{u \mid u \in Q, \bar{f}(u)=v \bar{\in} Q\}=\varnothing$; (8)和(9)式变成

$$
\begin{aligned}
Q^{*} & =Q^{\prime} \bigcup\{v \mid v \in \mathscr{D}, v \bar{\in} Q, f(v)=u \in Q\} \\
& =(Q-\{u \mid u \in Q, \bar{f}(u)=v \bar{\in} Q\}) \bigcup\{v \mid v \in \mathscr{D}, v \bar{\in} Q, f(v)=u \in Q\} \\
& =Q \bigcup\{v \mid v \in \mathscr{D}, v \bar{\in} Q, f(v)=u \in Q\}=Q^{\circ} ;
\end{aligned}
$$

(11)和(12)式分别成为 


$$
(R, \mathscr{F}) \circ\left(Q^{*}\right)=\bigcup[u]=\left\{u \mid u \in \mathscr{O},[u] \subseteq Q^{*}\right\}=\left\{u \mid u \in \mathscr{O},[u] \subseteq Q^{\circ}\right\}=\bigcup[u]=(R, F) \circ\left(Q^{\circ}\right)
$$

和

$$
(R, \mathscr{F})^{\circ}\left(Q^{*}\right)=\bigcup[u]=\left\{u \mid u \in \mathscr{O},[u] \cap Q^{*} \neq \varnothing\right\}=\left\{u \mid u \in \mathscr{O},[u] \cap Q^{\circ} \neq \varnothing\right\}=\bigcup[u]=(R, F)^{\circ}\left(Q^{\circ}\right),
$$

因此

$$
\left((R, \mathscr{F}) \circ\left(Q^{*}\right),(R, \mathscr{F})^{\circ}\left(Q^{*}\right)\right)_{\bar{F}=\varnothing}=\left((R, F) \circ\left(Q^{\circ}\right),(R, F)^{\circ}\left(Q^{\circ}\right)\right) .
$$

定理 2 函数双向 S-粗集与函数单向 S-粗集对偶满足

$$
\left((R, \mathscr{F}) \circ\left(Q^{*}\right),(R, \mathscr{F})^{\circ}\left(Q^{*}\right)\right)_{F=\varnothing}=\left((R, \bar{F}) \circ\left(Q^{\prime}\right),(R, \bar{F})^{\circ}\left(Q^{\prime}\right)\right) \text {. }
$$

定理 3 函数双向 S-粗集与函数粗集满足

$$
\left((R, \mathscr{F}) \circ\left(Q^{*}\right),(R, \mathscr{F})^{\circ}\left(Q^{*}\right)\right)_{\mathscr{F}=\varnothing}=\left(R_{-}(Q), R^{-}(Q)\right) .
$$

定理 4 函数单向 $\mathrm{S}$-粗集与函数粗集满足

$$
\left((R, F) \circ\left(Q^{\circ}\right),(R, F)^{\circ}\left(Q^{\circ}\right)\right)_{F=\varnothing}=\left(R_{-}(Q), R^{-}(Q)\right) .
$$

定理 5 函数单向 $\mathrm{S}$-粗集对偶与函数粗集满足

$$
\left((R, \bar{F})_{\circ}\left(Q^{\prime}\right),(R, \bar{F})^{\circ}\left(Q^{\prime}\right)\right)_{\bar{F}=\varnothing}=\left(R_{-}(Q), R^{-}(Q)\right) .
$$

推论 1 函数双向 S-粗集退化成函数粗集，必有

$$
\operatorname{As}\left(Q^{*}\right)=\varnothing \text {. }
$$

事实上，函数双向 $\mathrm{S}$-粗集退化成函数粗集，或者 $\left((R, \mathscr{F}) \circ\left(Q^{*}\right),(R, \mathscr{F})^{\circ}\left(Q^{*}\right)\right)=\left(R_{-}(Q)\right.$, $\left.R^{-}(Q)\right)$, 必有 $\mathscr{F}=\varnothing$ 或者 $F=\varnothing, \bar{F}=\varnothing$; 因此(15)式

$$
A s\left(Q^{*}\right)=\{u \mid v \in \mathscr{D}, v \bar{\in} Q, f(v)=u \tilde{\in} Q \text { 和 } u \in Q, \bar{f}(u)=v \in Q\}=\varnothing .
$$

推论 2 函数单向 S-粗集退化成函数粗集, 必有

$$
\operatorname{As}\left(Q^{\circ}\right)=\varnothing \text {. }
$$

推论 3 函数单向 S-粗集对偶退化成函数粗集, 必有

$$
A s\left(Q^{\prime}\right)=\varnothing \text {. }
$$

由定理 1 5, 推论 1 3, 容易证明命题 1 4.

命题 1 函数双向 S-粗集是函数单向 S-粗集的一般形式, 函数单向 S-粗集是函数双向 S-粗 集的特例.

命题 2 函数双向 S-粗集是函数单向 S-粗集对偶的一般形式, 函数单向 S-粗集对偶是函数 双向 S-粗集的特例.

命题 3 函数单向 S-粗集是函数粗集的一般形式，函数粗集是函数单向 S-粗集的特例.

命题 4 函数单向 S-粗集对偶是函数粗集的一般形式, 函数粗集是函数单向 S-粗集对偶的 特例.

函数 S-粗集的更多概念与特性, 见文献 [1 9]. 在第 5 节的讨论中, $R$-函数等价类 $[u]$ 和规 律 $[u]$ 两个概念不加区分, 直接使用. 


\section{5 规律的属性特征与规律生成}

[u] 是具有属性集 $\alpha=\left\{\alpha_{1}, \alpha_{2}, \cdots, \alpha_{k}\right\} \subset V$ 的规律, 若存在属性 $\beta \in V, \beta \bar{\in} \alpha$, 元素迁移 [8 14] $f \in F$ 把 $\beta$ 变成 $f(\beta)=\alpha^{\prime} \in \alpha$, 称 $[u]^{f}$ 是 $[u]$ 的 $f$-生成规律, 显然有

$$
\begin{gathered}
\operatorname{card}\left([u]^{f}\right) \leqslant \operatorname{card}([u]), \\
\operatorname{card}(\alpha) \leqslant \operatorname{card}\left(\alpha^{f}\right),
\end{gathered}
$$

这里 $\alpha^{f}=\alpha \bigcup\left\{f(\beta)=\alpha^{\prime}\right\}$ 是 $[u]^{f}$ 的属性集, $\operatorname{card}([u])$ 是 $[u]$ 的基数, $V$ 是属性论域.

[u] 是具有属性集 $\alpha=\left\{\alpha_{1}, \alpha_{2}, \cdots, \alpha_{k}\right\} \subset V$ 的规律, 若存在属性 $\alpha_{i} \in \alpha, i \in(1,2, \cdots, \lambda, \lambda<k)$, 元素迁移 ${ }^{[8 \sim 14]} \bar{f} \in \bar{F}$ 把 $\alpha_{i}$ 变成 $\bar{f}\left(\alpha_{i}\right)=\beta_{i} \bar{\in} \alpha$, 称 $[u]^{\bar{f}}$ 是 $[u]$ 的 $\bar{f}$-生成规律. 显然有

$$
\begin{gathered}
\operatorname{card}([u]) \leqslant \operatorname{card}\left([u]^{\bar{f}}\right), \\
\operatorname{card}\left(\alpha^{\bar{f}}\right) \leqslant \operatorname{card}(\alpha),
\end{gathered}
$$

这里 $\alpha^{\bar{f}}=\alpha-\left\{\bar{f}\left(\alpha_{i}\right)=\beta_{i}\right\}$ 是 $[u]^{\bar{f}}$ 的属性集.

下面讨论 $[u]$ 的规律生成.

给定规律 $[u]=\left\{u_{1}, u_{2}, \cdots, u_{m}\right\}, \hat{u}_{i}$ 是 $u_{i} \in[u]$ 的离散数据分布,如果

$$
\hat{u}_{i}=\left\{u_{i, 1}, u_{i, 2}, \cdots, u_{i, n}\right\},
$$

这里 $u_{i, k} \in \mathbb{R}, i=1,2, \cdots, m, k=1,2, \cdots, n ; \quad \mathbb{R}$ 是实数集.

利用

$$
u_{k}^{*}=\sum_{i=1}^{m} \hat{u}_{i, k}, \hat{u}_{i, k} \in \hat{u}_{i}, k=1,2, \cdots, n
$$

得到 $[u]$ 的合成规律 $[u]^{*}$, 为了简化又不引起误解, $[u]^{*}$ 仍记作 $[u]$; (16)式给出 $[u]\left([u]=[u]^{*}\right)$ 的 离散数据分布的数据点形式, 而且.

$$
\left(x_{1}, y_{1}\right),\left(x_{2}, y_{2}\right), \cdots,\left(x_{n}, y_{n}\right) .
$$

利用(16)式与 Lagrange 插值, 有

$$
p(x)=\sum_{j=1}^{n} y_{j} \prod_{\substack{i, j=1 \\ i \neq j}}^{n}\left(\frac{x-x_{i}}{x_{j}-x_{i}}\right),
$$

得到 $[u]\left([u]=[u]^{*}\right)$ 生成的多项式规律 $p(x)$, 而且

$$
p(x)=a_{n-1} x^{n-1}+a_{n-2} x^{n-2}+\cdots+a_{1} x+a_{0} .
$$

事实上, (18)式中的 $p(x)$ 是 $[u]\left([u]=[u]^{*}\right)$ 的生成形式之一, 其他生成形式略.

利用上面的概念，容易证明定理 6 和 7, 推论 4 和 5;

定理 6 若 $p(x)$ 是 $[u]$ 生成的规律, $p(x)^{f}$ 是 $[u]^{f}$ 生成的规律, 则

$$
\text { DIS }\left(p(x)^{f}, p(x)\right) \text {. }
$$


定理 7 若 $p(x)$ 是 $[u]$ 生成的规律, $p(x)^{\bar{f}}$ 是 $[u]^{\bar{f}}$ 生成的规律, 则

$$
\text { DIS }\left(p(x)^{\bar{f}}, p(x)\right) \text {, }
$$

这里DIS = discernibility 15,16$]$

推论 4 若 $[u]$ 的属性集 $\alpha$ 与 $[u]^{f}$ 的属性集 $\alpha^{f}$ 满足 $\alpha=\alpha^{f}-\left\{\bar{f}\left(\alpha^{\prime}\right)\right\}$, 则必有

$$
\operatorname{IND}\left(p(x), p(x)^{f}\right) \text {. }
$$

事实上, $p(x)^{f}$ 的属性集是 $\alpha^{f}=\alpha \bigcup\left\{f(\beta)=\alpha^{\prime}\right\}$, 这里: $\beta \in V, \beta \bar{\in} \alpha$. 元素迁移 $f \in F$ 把 $\beta$ 变成 $f(\beta)=\alpha^{\prime} \in \alpha$; 元素迁移 $\bar{f} \in \bar{F}$ 把 $\alpha^{\prime}$ 变成 $\bar{f}\left(\alpha^{\prime}\right)=\beta \bar{\in} \alpha$; 则有 $\alpha=\alpha^{f}-\left\{\bar{f}\left(\alpha^{\prime}\right)=\beta\right\}$, 在 这个条件下, $p(x)=p(x)^{f} ; p(x)$ 与 $p(x)^{f}$ 不可分辨 $[15,16]$.

推论 5 若 $[u]$ 的属性集 $\alpha$ 与 $[u]^{\bar{f}}$ 的属性集 $\alpha^{\bar{f}}$ 满足 $\alpha=\alpha^{\bar{f}} \cup\left\{f\left(\beta_{i}\right)\right\}$, 则必有

$$
\operatorname{IND}\left(p(x), p(x)^{\bar{f}}\right) \text {. }
$$

事实上, $p(x)^{\bar{f}}$ 的属性集是 $\alpha^{\bar{f}}=\alpha-\left\{\bar{f}\left(\alpha_{i}\right)=\beta_{i}\right\}$, 这里 $\alpha_{i} \in \alpha$. 元素迁移 $\bar{f} \in \bar{F}$ 把 $\alpha_{i}$ 变成 $\bar{f}\left(\alpha_{i}\right)=\beta_{i} \bar{\in} \alpha$, 元素迁移 $f \in F$ 把 $\beta_{i}$ 变成 $f\left(\beta_{i}\right)=\alpha_{i} \in \alpha$, 则有 $\alpha=\alpha^{\bar{f}} \cup\left\{f\left(\beta_{i}\right)=\alpha_{i}\right\}$, 在这个 条件下, $p(x)=p(x)^{\bar{f}} ; p(x)$ 与 $p(x)^{\bar{f}}$ 不可分辨 ${ }^{[15,16]}$. 这里 IND $=$ indiscernibility ${ }^{[15,16]}$.

\section{6 规律辨识准则与规律辨识应用}

\section{1 规律辨识准则}

$[Q]$ 上的任意两个规律 $[u]_{i}$ 与 $[u]_{j}(i \neq j)$, 如果它们的规律距离 $D\left([u]_{i},[u]_{j}\right)_{i \neq j}$ 满足

$$
D\left([u]_{i},[u]_{j}\right)_{i \neq j} \neq 0,
$$

则 $[u]_{j}$ 关于 $[u]_{i}$ 可辨识.

这里 $D\left([u]_{i},[u]_{j}\right)_{i \neq j}$ 是规律 $[u]_{i}$ 与规律 $[u]_{j}$ 的距离 $[9]$,

$$
D\left([u]_{i},[u]_{j}\right)_{i \neq j}=\frac{1}{n}\left(\sum_{i, j=0}^{n-1}\left(b_{j}-a_{i}\right)^{2}\right)^{1 / 2},
$$

其中 $b_{j}$ 是 $[u]_{j}$ 生成的规律 $p(x)_{j}=b_{n-1} x^{n-1}+b_{n-2} x^{n-2}+\cdots+b_{1} x+b_{0}$ 中的系数, $j=0,1,2, \cdots, n-1$; $a_{i}$ 是 $[u]_{i}$ 生成的规律 $p(x)_{i}=a_{n-1} x^{n-1}+a_{n-2} x^{n-2}+\cdots+a_{1} x+a_{0}$ 的系数, $i=0,1,2, \cdots, n-1$.

显然, 若 $D\left([u]_{i},[u]_{j}\right)_{i \neq j}=0$, 则 $[u]_{j}$ 关于 $[u]_{i}$ 不可辨识, $[u]_{i},[u]_{j} \in[\mathcal{Q}], i \neq j$.

\section{2 规律辨识定理}

定理 8(规律的 $F$-辨识定理) 规律 $[u]_{j}$ 关于规律 $[u]_{i}$ 可辨识的充分必要条件是函数迁移族 $F$ 满足

$$
F \neq \varnothing
$$


这里 $[u]_{i},[u]_{j} \in[\mathcal{U}], i \neq j$.

证明 1) 给定规律 $[u]_{i} \in[\mathcal{Q}],[u]_{i}=\left\{u_{i, 1}, u_{i, 2}, \cdots, u_{i, m}\right\}$; 因为 $(20)$ 式中 $F \neq \varnothing$, 而且存在 $v \in \mathscr{R}, v \bar{\in}[u]_{i}, f \in F$ 把 $v$ 变成 $f(v)=u^{\prime} \in[u]_{i}$, 则 $[u]_{i}$ 变成 $[u]_{j}=[u]_{i} \cup\left\{f(v)=u^{\prime}\right\}=\left\{u_{i, 1}, u_{i, 2}\right.$, $\left.\cdots, u_{i, m}, u^{\prime}\right\}=\left\{u_{i, 1}, u_{i, 2}, \cdots, u_{i, m}, u_{i, m+1}\right\}$; 故有 $[u]_{i}$ 和 $[u]_{j}$ 的合成规律的离散数据分布 $[u]_{i}^{*}$ 和 $[u]_{j}^{*}$. 设 $p(x)_{i}^{*}=a_{n-1} x^{n-1}+a_{n-2} x^{n-2}+\cdots+a_{1} x+a_{0}$ 是 $[u]_{i}^{*}$ 生 成 的多 项 式规律 ; $p(x)_{j}^{*}=b_{n-1} x^{n-1}+$ $b_{n-2} x^{n-2}+\cdots+b_{1} x+b_{0}$ 是 $[u]_{j}^{*}$ 生成的多项式规律，由第 5 节中得到的 $a_{n-1} \leqslant b_{n-1}, a_{n-2} \leqslant b_{n-2}, \cdots$, $a_{1} \leqslant b_{1}, a_{0} \leqslant b_{0} ; p(x)_{i}^{*}$ 与 $p(x)_{j}^{*}$ 满足规律辨识准则, 或者

$$
D\left([u]_{i},[u]_{j}\right)_{i \neq j} \neq 0,
$$

则 $[u]_{j}$ 关于 $[u]_{i}$ 可辨识.

2) 若 $[u]_{j}$ 关于 $[u]_{i}$ 可辨识, 或者

$$
D\left([u]_{i},[u]_{j}\right)_{i \neq j} \neq 0,
$$

使得上式成立，则必有 $F \neq \varnothing$.

定理 9(规律的 $\bar{F}$-辨识定理) 规律 $[u]_{j}$ 关于规律 $[u]_{i}$ 可辨识的充分必要条件是函数迁移族 $\bar{F}$ 满足

$$
\bar{F} \neq \varnothing,
$$

这里 $[u]_{i},[u]_{j} \in[\mathcal{Q}], i \neq j$.

定理 10(规律辨识无效定理) 规律 $[u]_{j}$ 关于规律 $[u]_{i}$ 辨识无效的充分必要条件是 $[u]_{j}$ 关于 $[u]_{i}$ 的规律距离 $D\left([u]_{i},[u]_{j}\right)_{i \neq j}$ 满足

$$
D\left([u]_{i},[u]_{j}\right)_{i \neq j}=0 .
$$

事实上, 若 $[u]_{j}$ 关于 $[u]_{i}$ 满足规律距离 $D\left([u]_{i},[u]_{j}\right)_{i \neq j}=0$, 则 $[u]_{j}$ 生成的多项式规律 $p(x)_{j}$ 与 $[u]_{i}$ 生成的多项式规律 $p(x)_{i}$ 满足

$$
p(x)_{j}=b_{n-1} x^{n-1}+b_{n-2} x^{n-2}+\cdots+b_{1} x+b_{0}=a_{n-1} x^{n-1}+a_{n-2} x^{n-2}+\cdots+a_{1} x+a_{0}=p(x)_{i},
$$

或者

$$
a_{n-1}=b_{n-1}, a_{n-2}=b_{n-2}, \cdots, a_{1}=b_{1}, a_{0}=b_{0},
$$

显然有

$$
\operatorname{IND}\left([u]_{j},[u]_{i}\right)_{i \neq j} .
$$

\section{3 规律辨识的应用}

这里给出一个应用例子, 它取自某信息传递系统 $\Omega$ 的一部分; 这个应用例子是在函数单 向 S-粗集上讨论的, 它是 $f$ - 规律辨识的应用; $\bar{f}$-规律辨识的应用在本节例子中略. 
设 $[u]$ 是系统 $\Omega$ 中的一个子系统, $[u]=\left\{u_{1}, u_{2}\right\} ; u_{1}$ 和 $u_{2}$ 是 $[u]$ 的两个输出规律(函数); 利 用第 5 节中的合成规律概念, 得到 $[u]$ 的合成输出规律 $p(x)$, 它是一个 7 次多项式, 而且

$$
\begin{aligned}
p(x)= & a_{7} x^{7}+a_{6} x^{6}+a_{5} x^{5}+a_{4} x^{4}+a_{3} x^{3}+a_{2} x^{2}+a_{1} x^{1}+a_{0} \\
= & -0.0055 x^{7}+0.1578 x^{6}-1.8419 x^{5}+11.2028 x^{4}-38.2424 x^{3} \\
& +73.1894 x^{2}-71.3602 x+29.0000 .
\end{aligned}
$$

(23)式表示系统 $[u]$ 处在正常工作状态, 子系统 [u] 的规律 $p(x)$ 与设定规律 $p(x)^{0}$ 一致, 而 且 $p(x)=p(x)^{0}$, 或者规律距离 $D\left(p(x), p(x)^{0}\right)=0$. 设定规律 $p(x)^{0}$ 的形式略.

在时刻 $t_{j} \in T$, 子系统 $[u]$ 受到不明规律 $v_{i} \bar{\in}[u]$ 的干扰与攻击, 使得 $[u]$ 的规律 $p(x)$ 变成 $p(x)^{f}$, 而且

$$
\begin{aligned}
p(x)^{f}= & b_{7} x^{7}+b_{6} x^{6}+b_{5} x^{5}+b_{4} x^{4}+b_{3} x^{3}+b_{2} x^{2}+b_{1} x^{1}+b_{0} \\
= & 0.0014 x^{7}-0.0275 x^{6}+0.1708 x^{5}-0.0792 x^{4}-3.1496 x^{3} \\
& +12.7067 x^{2}-16.8226 x+10.8000 .
\end{aligned}
$$

在时刻 $t_{j} \in T,(23)$ 与(24)式满足规律辨识准则, 或者

$$
D\left(p(x), p(x)^{f}\right) \neq 0 .
$$

子系统 [u] 的规律在 $t_{j} \in T$ 时刻被辨识, 或者时刻 $t_{j} \in T, p(x)^{f} \neq p(x)$. 时刻 $t_{j} \in T$, 子系统 [u] 的实际表现为传送的图像混乱、失真.

利用(23)和(24)式, 应该找到不明规律 $v_{i}$. 把(23)和(24)式分别离散化, 并以数据点的形式 表示如下:

$$
\begin{gathered}
p(x):\left(1, y_{1}\right),\left(2, y_{2}\right),\left(3, y_{3}\right),\left(4, y_{4}\right),\left(5, y_{5}\right),\left(6, y_{6}\right),\left(7, y_{7}\right),\left(8, y_{8}\right), \\
p(x)^{f}:\left(1, y_{1}^{f}\right),\left(2, y_{2}^{f}\right),\left(3, y_{3}^{f}\right),\left(4, y_{4}^{f}\right),\left(5, y_{5}^{f}\right),\left(6, y_{6}^{f}\right),\left(7, y_{7}^{f}\right),\left(8, y_{8}^{f}\right) .
\end{gathered}
$$

(25)和(26)式的具体数据略.

由(25)和(26)式及 $\Delta y_{i}=y_{i}^{f}-y_{i}(i=1,2, \cdots, 8)$, 得到数据点

$$
v(x):(1,1.5),(2,2.6),(3,3.7),(4,4),(5,6),(6,8),(7,0.8),(8,3) .
$$

由(17)和(27)式, 得到

$$
\begin{aligned}
v(x)= & c_{7} x^{7}+c_{6} x^{6}+c_{5} x^{5}+c_{4} x^{4}+c_{3} x^{3}+c_{2} x^{2}+c_{1} x^{1}+c_{0} \\
= & 0.0068 x^{7}-0.1853 x^{6}+2.0128 x^{5}-11.2819 x^{4}+35.0928 x^{3} \\
& -60.4828 x^{2}+54.5376 x-18.2000 .
\end{aligned}
$$

(28)式是入侵到子系统 $[u]$ 中的干扰与攻击规律 $v$. 启动子系统 $[u]$ 中的智能识别与跟踪过滤装 置, 对 $v(x)$ 给予阻隔. 因为一些原因, 本文省略对 $p(x)^{f}$ 的智能识别与 $v(x)$ 的跟踪过滤装置的 结构.

这个例子表明: $v(x) \bar{\epsilon}[u(x)], f \in F$ 把 $v(x)$ 变成 $f(v(x))=u(x)^{\prime} \in[u(x)]$, 使得子系统 
$[u]=\left\{u_{1}, u_{2}\right\}$ 变成 $[u]^{f}=[u] \bigcup\{f(v(x))\}=\left\{u_{1}, u_{2}, f(v(x))=u_{3}\right\}=\left\{u_{1}, u_{2}, u_{3}\right\}$, 而且使得

$$
D\left([u],[u]^{f}\right) \neq 0 \text {. }
$$

(29)式说明子系统的输出规律 $p(x)$ 被攻击, $p(x)$ 偏离了设定规律而被辨识. 函数 $\mathrm{S}$-粗集对于 这个信息传递系统的规律辨识的其他应用略.

\section{7 元素迁移与函数迁移}

这里给出元素迁移和函数迁移的概念, 这些概念对于接收本文第 1 5 节中的讨论是重要 的.

给定 $R$-元素等价类 $[x]=\left\{x_{1}, x_{2}, \cdots, x_{m}\right\} \subset U, Y=\left\{y_{1}, y_{2}, \cdots, y_{m}\right\}$ 是 $[x]$ 的特征值集合, $y_{i}$ 是 $x_{i}$ 的特征值, $i=1,2, \cdots, m ;[a, b]$ 是 $Y$ 生成的特征值区间, 而且

$$
\begin{aligned}
& a=\min _{i=1}^{m}\left(y_{i}\right), \\
& b=\max _{j=1}^{m}\left(y_{j}\right) ; y_{j} \in \mathbb{R},
\end{aligned}
$$

$\mathbb{R}$ 是实数集.

显然, 对于元素 $x_{i}, x_{j} \in[x]$, 有元素 $x_{i}$ 和 $x_{j}$ 的特征值 $y_{i}, y_{j} \in[a, b]$; 对于元素 $x_{p}, x_{q} \in U$, $x_{p}, x_{q} \bar{\in}[x]$, 有 $x_{p}$ 和 $x_{q}$ 的特征值 $y_{p}, y_{q} \bar{\in}[a, b]$. 若存在变换 $f \in F$, 把 $y_{p}$ 和 $y_{q}$ 变成 $f\left(y_{p}\right)$, $f\left(y_{q}\right) \in[a, b]$, 则有 $x_{p}, x_{q} \in[x]$; 或者

$$
x_{p}, x_{q} \in U, \quad x_{p}, x_{q} \bar{\epsilon}[x] \Rightarrow f\left(x_{p}\right), f\left(x_{q}\right) \in[x],
$$

其中变换 $f \in F$ 是元素迁移.

对于元素 $x_{\lambda} \in[x], y_{\lambda}$ 是 $x_{\lambda}$ 的特征值, 若存在变换 $\bar{f} \in \bar{F}$ 把 $y_{\lambda} \in[a, b]$ 变成 $\bar{f}\left(y_{\lambda}\right) \bar{\epsilon}[a, b]$, 则有 $x_{\lambda} \bar{\epsilon}[x]$; 或者

$$
x_{\lambda} \in[x] \Rightarrow \bar{f}\left(x_{\lambda}\right)=u_{\lambda} \bar{\epsilon}[x]
$$

其中变换 $\bar{f} \in \bar{F}$ 是元素迁移.

给定 $R$-函数等价类 $[u(x)] \subset \mathscr{O},[u(x)]=\left\{u(x)_{1}, u(x)_{2}, \cdots, u(x)_{m}\right\} ;\left[a_{i}, b_{i}\right]$ 是 $u(x)_{i} \in[u(x)]$ 的 定义域, $a_{i} \leqslant b_{i} ; a_{i}, b_{i} \in \mathbb{R}, i=1,2, \cdots, m .\left[c_{i}, d_{i}\right]$ 是 $u(x)_{i} \in[u(x)]$ 的值域, $c_{i} \leqslant d_{i} ; c_{i}, d_{i} \in \mathbb{R} . R-$ 函数等价类 $[u(x)]$ 的定义域和值域分别是 $[a, b]$ 和 $[c, d], a \leqslant b, c \leqslant d, a, b, c, d \in \mathbb{R}$; 而且

$$
\begin{aligned}
& a=\min _{i=1}^{m}\left(a_{i}\right), \quad b=\max _{i=1}^{m}\left(b_{i}\right), \\
& c=\min _{i=1}\left(c_{i}\right), \quad d=\max _{i=1}^{m}\left(d_{i}\right) .
\end{aligned}
$$

若 $u(x)_{j} \in[u(x)]$, 则 $x \in[a, b], u(x)_{j} \in[c, d]$; 若 $u(x)_{p} \bar{\in}[u(x)]$, 则 $x \bar{\in}[a, b], u(x)_{p} \bar{\epsilon}[c, d]$. 存在变换 $f \in F$, 对于 $v(x) \bar{\in}[u(x)], f \in F$ 使得 $v(x)$ 中的 $x \in[a, b], f(v(x)) \in[c, d]$, 则有 $f(v(x))$ 
$=u(x) \in[u(x)], f \in F$ 是函数迁移, 或者

$$
\exists v(x) \in \mathscr{O}, v(x) \bar{\epsilon}[u(x)] \Rightarrow f(v(x))=u(x) \in[u(x)] .
$$

反之, 存在 $u(x)_{j} \in[u(x)], \bar{f} \in \bar{F}$ 使得 $u(x)_{j}$ 中 的 $x \bar{\in}[a, b], \bar{f}\left(u(x)_{j}\right) \bar{\epsilon}[c, d]$, 则有 $\bar{f}\left(u(x)_{j}\right)=v(x)_{j} \bar{\epsilon}[u(x)] ; \bar{f} \in \bar{F}$ 是函数迁移, 或者

$$
\exists u(x)_{j} \in[u(x)] \Rightarrow \bar{f}\left(u(x)_{j}\right)=v(x)_{j} \bar{\epsilon}[u(x)] .
$$

直观地说, 元素迁移 $f \in F$ 是把 $[x]$ 外的元素 $x$ 迁移到 $[x]$ 内, $\bar{f} \in \bar{F}$ 是把 $[x]$ 内的元素 $x$ 迁 移到 $[x]$ 外. 函数迁移 $f \in F$ 是把 $[u(x)]$ 外的函数 $v(x)$ 迁移到 $[u(x)]$ 内; $\bar{f} \in \bar{F}$ 是把 $[u(x)]$ 内的 函数 $u(x)$ 迁移到 $[u(x)]$ 外. 元素迁移 $f \in F, \bar{f} \in \bar{F}$; 函数迁移 $f \in F, \bar{f} \in \bar{F}$ 是一类简单的函数 族.

\section{8 讨论}

波兰数学家Pawlak 1982 年提出粗集(rough sets) ${ }^{[15,16]}$, 给出粗集结构与粗集的应用; 这一 杰出的学术成就, 使得众多的理论与应用学者对粗集产生兴趣. Pawlak Z粗集是以静态 $R$-元素 等价类 $[x]$ 定义的, Pawlak Z粗集是一个静态意义下的粗集, 因此, Pawlak Z粗集的应用范围受 到了限制. 2002 年, 文献 [10]改进了 Pawlak Z粗集, 提出S-粗集(singular rough sets), S-粗集是以 动态 $R$-元素等价类 $[x]$ 定义的, $\mathrm{S}$-粗集具有了动态特性, 动态特性来自元素迁移; 文献 [8,9,11 14]对S-粗集给出了进一步讨论. S-粗集推广了Pawlak Z粗集，拓宽了Pawlak Z粗集的应 用范围. 利用Pawlak Z粗集, S-粗集对于信息系统中的规律辨识和规律挖掘的研究遇到了困难, 因为Pawlak Z粗集和S-粗集不具有规律特征. 什么是规律? 一个函数是一个规律. 2005 年, 文 献 [1,2]改进了S-粗集, 提出函数S-粗集和函数粗集; 给出它们的结构; 函数粗集是函数 S-粗集 的静态形式. 文献 [3 9]对函数 S-粗集给出了进一步讨论. 函数 S-粗集是以具有动态特 性的 $R$-函数等价类 $[u]$ 定义的; 函数粗集是以具有静态特性的 $R$-函数等价类 $[u]$ 定义的. 函数 $\mathrm{S}$-粗集推广了 $\mathrm{S}$-粗集, 拓宽了 S-粗集的应用范围. 从数学结构和静态-动态的双重意义下看, Pawlak Z粗集是 S-粗集的特例, S-粗集是函数S-粗集的特例; 反之, 函数 S-粗集是S-粗集的一般 形式, S-粗集是Pawlak Z粗集的一般形式. 这里应该特别指出: S-粗集改进了Pawlak Z粗集，函 数S-粗集改进了S-粗集, 这些改进没有改变Pawlak Z粗集中的等价关系 $R^{[9]}$.

信息系统的输出规律在时间区间 $\left[t_{1}, t_{k}\right]$ 上是一个函数(多端输出是时间区间 $\left[t_{1}, t_{k}\right]$ 上的多 个函数), 输出规律与设定规律之间产生偏差, 导致信息系统的输出规律的漂移, 使得信息系 统输出规律远离规律零点, 由此产生对信息系统输出规律辨识的研究. 函数 S-粗集为信息系 统中规律辨识研究提供了新的研究思想与研究工具.

函数 S-粗集与信息系统中的规律辨识进行交叉、融合、渗透，这将成为信息系统中规律辨 识研究的一个新方向. 


\section{参考文献}

1 Shi K Q. Function S-rough sets and function transfer. An Int J Adv Syst Sci Appl, 2005, 5(1): 1—8

2 史开泉. 函数 S-粗集. 山东大学学报(理学版), 2005, 40(1): 1-10

3 Zhang P, Shi K Q. Function S-rough sets and rough law heredity-mining. In: IEEE Proc 4th Int Conf Mach Learn Cybern, 2005, 7(3): 3148-3152 [DOI]

4 Shi K Q, Yao B X. Function S-rough sets and recognition of financial risk laws. In: 1st Int Conf Rough Sets Knowl Technol, 2006, 1(1): 247-253 [DOI]

5 Wang H Y, Shi K Q. Function S-rough sets and investment warning estimation. Int J Fuzzy Math, 2007, 14(1): 155-168

6 Cui Y Q, Shi K Q. Function S-rough sets and its applications. J Syst Eng Electron, 2006, 17(2): 331 — $338 \underline{\text { [DOI] }}$

7 Shi K Q, Xia J R. Function S-rough sets and mining-discovery of rough law in systems. J Syst Eng Electron, 2006, 17(4): 919-926 [DOI]

8 史开泉, 崔玉泉. S-粗集与粗决策. 北京: 科学出版社, 2006. 39-54

9 史开泉, 姚炳学. 函数 S-粗集与系统规律挖掘. 北京:科学出版社, 2007. 51-68

10 Shi K Q. S-rough sets and its applications in diagnosis-recognition for disease. In: IEEE Proc 1st Int Conf Mach Learn Cybern, 2002, 4(1): 50-54

11 Shi K Q. S-rough sets and knowledge separation. J Syst Eng Electron, 2005, 16(2): 403-410

12 Shi K Q, Chang T C. One direction S-rough sets. Int J Fuzzy Math, 2005, 13(2): 319-334

13 Shi K Q. Two direction S-rough sets. Int J Fuzzy Math, 2005, 13(2): 335-349

14 Shi K Q, Cui Y Q. F-decomposition and $\bar{F}$-reduction of S-rough sets. An Int J Adv Syst Sci Appl, 2004, 4(4): 487-499

15 Pawlak Z. Rough sets. Int J Comput Inform Sci, 1982, 32(11): 341-356

16 Pawlak Z. Rough classification. Int J Man-Machine Stud, 1984, 27(20): 469-483 